\title{
Temporary Balloon Occlusion of the Internal Iliac Arteries to Prevent Massive Hemorrhage during Cesarean Delivery in a Patient with Placenta Previa Increta - Case Report
}

\author{
Krzysztof Pyra $^{1 *}$, Lukasz Światlowski ${ }^{1}$, Marek Grzechnik ${ }^{2}$, Piotr Dziduch ${ }^{2}$, Maryla Kuczyńska ${ }^{1}$, \\ Anna Drelich-Zbroja ${ }^{1}$, Tomasz Jargiełlo ${ }^{1}$ \\ ${ }^{I}$ Department of Interventional Radiology and Neuroradiology, Medical University of Lublin, Poland \\ ${ }^{2}$ Chair and Department of Obstetrics and Perinatology, Medical University of Lublin, Poland
}

*Corresponding Author: Krzysztof Pyra, Department of Interventional Radiology and Neuroradiology, Medical University of Lublin, Slawinkowska 63, 20-810 Lublin, Poland, Email: k.pyra@ poczta.fm

\begin{abstract}
Objective: The number of cesarean deliveries has been constantly increasing, leading to higher incidence of complications due to abnormal placental location. Placenta accreta and previa constitute the major cause of severe peripartum bleeding - still posing a challange in the obstetric practice. Cooperation between the gynecologist and the interventional radiologist creates a good solution what will be presented in the case below.

Case Report: A 37-year-old patient, after 2 caesareans, with placenta percreta with possible bladder invasion was admitted to hospital in $37^{\text {th }}$ week of pregnancy. Due to high risk of hemorrhage temporary balloon occlusion of the internal iliac arteries in hybrid room was performed. After delivering the fetus massive bleeding occurred, balloon inflation was necessary to temporarily block the main blood inflow. Intraoperative bleeding subsided and hysterectomy was continued in dry operation field. Intraoperative blood loss was only $840 \mathrm{ml}$. No complications were observed during postpartum period.
\end{abstract}

Discussion: Prophylactic iliac arteries balloon catheterization with their temporary occlusion enables intraoperative blood loss reduction and relatively comfortable working environment for the surgeon.

Keywords: Placenta increta, bleeding, delivery, hemorrhage, embolization, balloon occlusion

\section{INTRODUCTION}

In recent years, the number of cesarean deliveries has been constantly increasing, leading to higher incidence of complications due to abnormal placental location or attachment in the following pregnancies (1), with an obstetric hemorrhage in the postpartum period being the most severe of them. Placenta increta is a serious obstetrical complication caused by a defect in the decidua basalis resulting in an abnormally invasive placental implantation. Several studies have shown that conservative management by leaving placenta in situ was successful in many cases with invasive placental implantation (2). But there were still a number of failed cases with delayed hysterectomy and severe maternal morbidity. Placenta accreta and previa are the two forms of placental maldevelopment, which constitute the major cause of severe peripartum bleeding - the most frequent cause of maternal mortality with an incidence rate reported as high as $30 \%$ (3-5). These two conditions still pose a challange in the obstetric practice (6). Case below is an interesting and informative on the basis of which we present diagnostic methods and a safe, minimally invasive way of delivery which is at risk of massive hemorrhage.

\section{Case Presentation}

A 37-year-old patient pregnant for the third time, with a record of 2 cesarean births, no symptoms and in overall good condition, was admitted to gynecology ward with a diagnosis of central placenta previa. Transvaginal ultrasound (TVUS) showed central placenta previa attached to the anterior uterine wall and previous caesarean scar. Magnetic resonance was performed in $37^{\text {th }}$ week of pregnancy, revealing placenta percreta with possible invasion of the 
bladder. Fetal development was consistent with the gestational age. The patient was informed about possible complications of the cesarean section, including total hysterectomy and potentially lethal massive hemorrhage in the postpartum period. Temporary balloon occlusion of the internal iliac arteries was planned as a preventive measure against bleeding.

The intervention was performed in a hybrid room under fluoroscopic guidance. Through bilateral femoral access, two $8 \mathrm{~mm}$ balloon catheters were introduced to the proximal internal iliac arteries under local anesthesia. The procedure was simultaneously conducted by two interventional radiologists to minimize the radiation dose. Control balloon inflation was performed to verify tightness of the sealing. The surgical part of the intervention was conducted then. A longitudinal cesarean section was made under general anesthesia to open the abdominal cavity. A classical longitudinal incision of the uterine body was then performed, through which the fetal head was exteriorized. A female live fetus was delivered from green amniotic fluid. Hysterectomy was undertaken due to macroscopically visible signs of placental invasion to the right iliac fossa within distal part of the uterus. Because of the massive bleeding, inflation of balloon catheters was necessary to temporarily block the main blood inflow. Intraoperative bleeding subsided and hysterectomy was continued in dry operation field. The uterus was removed in a typical manner without adnexectomy. The balloons were then deflated, allowing for reperfusion. By the end of operation, hemostasis control was performed and fibrin sealent patch (TachoSil) was placed to cover deperitonealized bladder surface. Safety drainage catheter was left in the abdominal cavity. The abdominal integument was sutured in layers. Intraoperative blood loss was $840 \mathrm{ml}$. 1 unit of packed red blood cells was administered to the patient during surgery.

Patient post-operative status was scored as 9/10 in Aldrete's system (scoring system is a commonly used scale for determining when people can be safely discharged from the postanesthesia care unit), with BP - 128/75 mmHg, HR - 71bpm, saturation 99\%. Control angiography was performed revealing no signs of active contrast extravasation. Patient was discharged home on day 6 with normal morphology. No complications were observed during postpartum period (6 weeks).

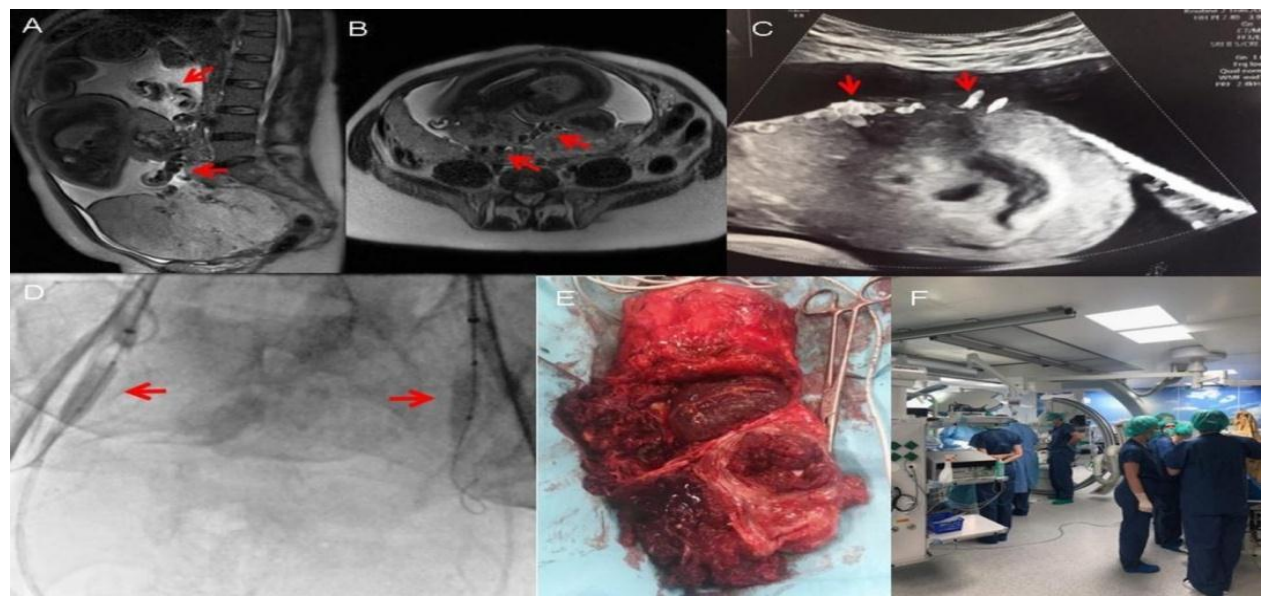

Figure: A, B. MRI examination: T2 sequences, sagittal (A) and transverse (B) projection. Vessels penetrating into the myometrium marked with red arrows. C. Real time Doppler Ultrasound: Vessels penetrating the lower part of the myometrium marked with red arrows - placenta accreta feature. D. Bilateral balloon catheters (markers indicate both ends of the balloons) are visible in the proximal internal iliac arteries. No contrast filling of the pelvic vessels is seen during control run after balloon inflation. Normal blood flow within external iliac arteries. Fetal head visible in the background [*]. E. Hysterectomy without appendages, significant part of the complete placenta praevia and accreta in the lower part of the uterus is visible. F. Hybrid room: Gynecologists on the left, neonatologist on the right.

\section{DISCUSSION}

Since placenta increta can cause life-threatening hemorrhage, disseminated intravascular coagulation (DIC), acute renal failure, infection, and so on, hysterectomy is the recommended form of management for most cases. Hysterectomy could not be avoided in this case. The frequency of hysterectomy in many of the published articles regarding labor in the presence of placenta increta varies from $36 \%$ to even $100 \%$ (7) 
The placenta was relatively heavy with macroscopic signs of myometrial invasion and previa. Histopathological examination verified the diagnosis of placenta previa increta. Cesarean hysterectomy for adherent placenta is associated with increased maternal morbidity due to massive hemorrhage requiring large volume blood transfusion, bladder or ureteric injury, intensive care unit (ICU) admission and prolonged hospital stay. Sumigama et al. reported the average blood loss of $3630-2216$ $\mathrm{mL}$ for placenta increta and 12,140 - $8343 \mathrm{~mL}$ for placenta percreta in traditional cesarean surgery, and one patient died of hemorrhage (8).

Prophylactic iliac arteries balloon catheterization with their temporary occlusion enables intraoperative blood loss reduction and relatively comfortable working environment for the surgeon. It reduces the incidence of major complications, need for blood components transfusion and is associated with better patient outcomes. In some even difficult cases, thanks to this method, if there is such a need, we are able to save the uterus. The case describes an alternate minimally invasive approach for cesarean hysterectomy.

\section{REFERENCES}

[1] Allahdin S, Voigt S, Htwe TT. Management of placenta praevia and accreta. J ObstetGynaecol 2011;31(1):1-6.
[2] Daney de Marcillac F, Lecointre L, Guillaume A, et al. [Maternal morbidity and mortality associated with conservative management for placenta morbidly adherent (accreta) diagnosed during pregnancy. Report of 15 cases]. J Gynecol

[3] Silver RM. Abnormal placentation placenta previa vasa previa, and placenta accreta. Obstet Gynecol2015;126:654-68

[4] Berg CJ, Atrash HK, Koonin LM. Pregnancyrelated mortality in the United States. Obstet Gynecol 1990;88:161e7.

[5] Hayes E, Ayida G, Crocker A. The morbidly adherent placenta: diagnosis and management options. Curr Opin Obstet Gynecol 2011;23(6):448e53.

[6] Royal College of Obstetricians and Gynaecologists. Placenta praevia, placenta praevia accreta, and vasa praevia: diagnosis and management: Green-top Guideline No. 27. Available from: https://www.rcog.org.uk/ globalassets/

[7] Eyal Sivan, Maya Spira, Reuven Achiron, Uri Rimon, Gil Golan, Shali Mazaki-Tovi, Eyal Schiff. Prophylactic Pelvic Artery Catheterization and Embolization in Women with Placenta Accreta: Can It Prevent Cesarean Hysterectomy? Amer J Perinatol 2010; 27(6): $455-461$

[8] Sumigama S, Itakura A, Ota T, et al. Placenta previa increta/percreta in Japan: a retrospective study of ultrasound findings management and clinical course. J Obstet Gynaecol Res. 2007;33:606e611.

Citation: Krzysztof Pyra, Łukasz Świattowski, Marek Grzechnik, Piotr Dziduch, Maryla Kuczyńska, Anna Drelich-Zbroja, Tomasz Jargielto. Temporary Balloon Occlusion of the Internal Iliac Arteries to Prevent Massive Hemorrhage during Cesarean Delivery in a Patient with Placenta Previa Increta-Case Report. ARC Journal of Gynecology and Obstetrics. 2019; 4(1):9-11. DOI:dx.doi.org/10.20431/2456-0561.0401002.

Copyright: (0) 2019 Authors. This is an open-access article distributed under the terms of the Creative Commons Attribution License, which permits unrestricted use, distribution, and reproduction in any medium, provided the original author and source are credited. 